\title{
Why Deep Neural Nets Cannot Ever Match Biological Intelligence and What To Do About It?
}

\author{
Danko Nikolić c $^{1,3}$ \\ ${ }^{1}$ DXC Technology, Frankfurt am Main, Germany \\ ${ }^{2}$ Frankfurt Institute for Advanced Studies (FIAS), Ruth-Moufang-Straße 1, D-60438 Frankfurt/M, Germany \\ ${ }^{3}$ Department of Psychology, Faculty of Humanities and Social Sciences, University of Zagreb, Croatia
}

\begin{abstract}
The recently introduced theory of practopoiesis offers an account on how adaptive intelligent systems are organized. According to that theory, biological agents adapt at three levels of organization and this structure applies also to our brains. This is referred to as tri-traversal theory of the organization of mind or for short, a T3-structure. To implement a similar T3-organization in an artificially intelligent agent, it is necessary to have multiple policies, as usually used as a concept in the theory of reinforcement learning. These policies have to form a hierarchy. We define adaptive practopoietic systems in terms of hierarchy of policies and calculate whether the total variety of behavior required by real-life conditions of an adult human can be satisfactorily accounted for by a traditional approach to artificial intelligence based on T2-agents, or whether a T3-agent is needed instead. We conclude that the complexity of real life can be dealt with appropriately only by a T3-agent. This means that the current approaches to artificial intelligence, such as deep architectures of neural networks, will not suffice with fixed network architectures. Rather, they will need to be equipped with intelligent mechanisms that rapidly alter the architectures of those networks.
\end{abstract}

Keywords: Artificial intelligence, neural networks, strong artificial intelligence, practopoiesis, machine learning

\section{Introduction: Hierarchy of policies}

Practopoiesis is a recent theory of how adaptive agents are organized and proposes a number of principles under which such systems operate ${ }^{[1]}$. One of the key presumptions of practopoiesis is that adaptive mechanisms are organized into a specific type of hierarchy: Mechanisms lower on the hierarchy determine the properties of the mechanisms higher on the hierarchy. Interactions among those levels of organization are described by concepts such as monitorand-act unit and cybernetic knowledge, and by principles such as knowledge extraction, knowledge shielding, downward pressure for adjustment, and equi-level interactions. It has been also proposed that practopoiesis has implications for development of machine learning and artificial intelligence $(\mathrm{AI})^{[1,2]}$.

Practopoietic systems can be described from the perspective of machine learning as follows. The entire set of adaptive capabilities of an organism (i.e., monitor-and-act units) at one level of organization, in the terminology of machine learning, can be described as the policy $(\pi)$ for generating actions. Similarly, cybernetic knowledge ${ }^{[1]}$ can be understood as an optimal policy of machine learning. Recent analyses showed that adaptations of neurons and neural

\footnotetext{
Research Article

Special Issue on Human-inspired Computing

Manuscript received January 16, 2017; accepted May 10, 2017; published online July 4, 2017

This work was supported by Hertie Foundation and Deutsche Forschungsgemeinschaft.

Recommended by Associate Editor Hong Qiao

(C) The Author(s) 2017
}

networks enhance computations ${ }^{[3,4]}$.

Importantly, a practopoietic agent may have different sets of policies, some of them acting on the environment but others acting on the agent itself. These sets form a hierarchy.

Thus, practopoietic hierarchy is an arrangement in which, for policy $x$, there is a policy $y$ whose actions change policy $x$. This makes it a T2-agent, due to the actions executed at two levels of organization. To indicate that actions of policy $y$ change policy $x$, we write:

$$
\pi_{y} \rightarrow \pi_{x}
$$

In that case, TD-learning ${ }^{[5]}$ and Q-learning algorithms ${ }^{[6]}$ are considered special policies belonging to $\pi_{y}$.

Importantly, however, according to practopoietic theory, biological T3-systems have also a third policy ${ }^{[1]}$ — referred to as tri-traversal theory of human cognition. The theory claims that there are fast and slow "learning" mechanisms and that the slow mechanisms train the fast ones. Thus, a full agent can be described then as

$$
\pi_{G} \rightarrow \pi_{A} \rightarrow \pi_{N}
$$

whereby, by following the tri-traversal theory, we presume that $\pi_{G}$ is stored in genes, $\pi_{A}$ in the rules for neural adaptation responsible for fast changes to the system (referred to also as anapoiesis), and $\pi_{N}$ in the properties of the neural network.

This relationship can be also described as fast learning (by operations of $\pi_{A}$ ) and slow learning how to learn fast (by operations of $\pi_{G}$ ). Here, most of the knowledge 
acquired through the lifetime of an agent is acquired in the properties of $\pi_{A}$, not in $\pi_{N}$ as would be the case in the most modern approaches to AI. In other words, T3-agents store knowledge in the rules on how to quickly learn.

To describe the interaction between an agent and its environment, we can write

$$
\pi_{G} \rightarrow \pi_{A} \rightarrow \pi_{N} \rightarrow \mathbf{U}
$$

where $\mathbf{U}$ stands for the surrounding world or Umwelt.

To describe the adaptive capabilities of an entire species, there is one additional policy, $\pi_{E}$, which determines the genome $\pi_{G}$. This policy operates according to the rules of evolution by natural selection. Thus, the adaptive structure of an entire species can be described as

$$
\pi_{E} \rightarrow \pi_{G} \rightarrow \pi_{A} \rightarrow \pi_{N} .
$$

These levels of adaptation in Tn-systems, would be distinguished from layers in the hierarchy of neural networks. Even if a network has 1000 layers, it remains to operate as a T1 system if there is no learning, and as T2 if a typical form of learning such as backpropagation is applied.

To create a T3 system, one needs to add a level at which the learning algorithm adapts. For example, an algorithm that uses feedback from the environment to adapt the learning rate of a gradient descent can be considered to contribute overall to a T3 organization of the entire system. This is because there are three levels of adaptation: network $<$ learning algorithm (teacher of the network) $<$ teacher of the learning algorithm (learning to learn). In the present paper, we are considering systems in which not only a small bit of knowledge is used at lower levels of organization to form a T3 system (e.g., only on parameter value, the learn- ing rate), but where the knowledge on how to learn contains a huge amount of information, i.e., the system becomes an expert on how to learn. See Fig. 1 for organization of biological minds according to the theory of practopoiesis.

Practopoiesis proposes that all living individuals have T3 organization, starting from each biological cell. Importantly, adding multiple Tn systems does not increase $n$. So, while each living cell alone operates as a T3 system, an entire organism built off billions of such cells remains to present also a T3 system. Similarly, a group of individual organisms or an entire society still form a T3 system.

The inclusion of evolution to form a T4 system applies to a single species and to the biosphere as a whole ${ }^{[1]}$. Thus, according to the theory, the entire evolving life on planet Earth can be understood as operating with four levels of policies, whereas each individual agent has "only" three.

\subsection{Generalizing actions}

In reinforcement learning theory, actions of an agent are conceptually different from the processes of learning by that agent. Practopoiesis generalizes all those forms of actions to one single concept: adaptive traverses (here, indicated by arrows, $\rightarrow$ ). A traverse is whenever knowledge of an agent together with the feedback from environment are used to make some changes either to the agent itself or to the environment. Two traverses are considered different if their mutual impacts are asymmetric: If one traverse affects the knowledge of the other but not the other way around. For example, a back propagation learning rule changes the mapping function of a neural net, but the mapping function of the neural net does not change the learning rule. Hence, here two traverses can be distinguished.

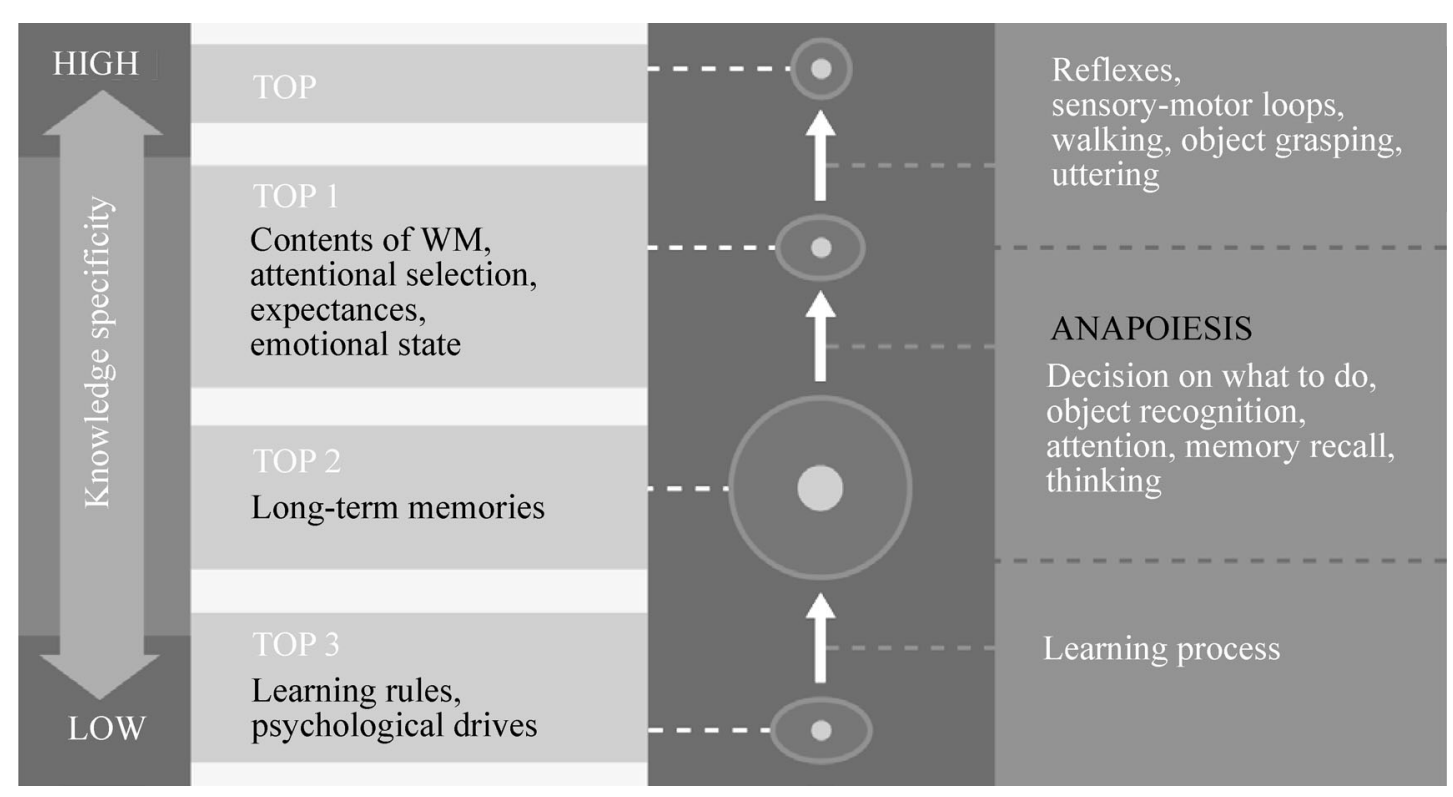

Fig. 1 The organization of biological minds according to the theory of practopoiesis. The descriptions of the three traverses are shown on the right. On the left described is the knowledge that each traverse uses for its operations but also the knowledge that each of the traverses creates by its operations. Top to Top-3 indicate the depths of adaptive levels. 
A system with operational capabilities at $n$ levels is a Tn-system and has $n$ traverses, each directed either towards the outside of the agent or towards inside of the agent (the latter being often referred to as learning).

The total number of traverses equals the number of organization levels at which policies exist. This is because actions of the policy at the top level of organization, $\pi_{N}$, affect the environment directly. Hence, the full interaction (with all the arrows) between a biological species as an agent and its environment can be written as

$$
\pi_{E} \rightarrow \pi_{G} \rightarrow \pi_{A} \rightarrow \pi_{N} \rightarrow \mathbf{U}
$$

That way reinforcement learning can be considered a special case of practopoietic systems. From the above considerations, we can also see that reinforcement learning is not nearly as sophisticated implementation of practopoiesis as is natural intelligence. The reason is more adaptive levels in natural intelligence.

\subsection{Generalizing feedback}

In reinforcement learning, learning mechanisms receive feedback. In practopoietic systems, policies at each level of organization receive feedback inputs too. These inputs are conceptually different in reinforcement learning theory: The input for $\pi_{x}$ is the identity of state $s$; the input for $\pi_{y}$ is the reward $r$.

Practopoiesis conceptually generalizes those feedback inputs as $i_{k}$, where $k$ is the level of organization of the system (E, G, A or N). Feedback inputs can be acquired through sensory inputs shared across policies at different levels. For example, a camera can provide the necessary information for $\pi_{N}$ and $\pi_{A}$.

The goal of the present study is to demonstrate the advantages of such hierarchical organization of multiple policies (traverses) as compared to an agent with fewer policies. The present analysis focuses on interactions $\pi_{A} \rightarrow \pi_{N} \rightarrow$ $\mathbf{U}$, presuming that the policies $\pi_{A}$ have been already put in place. The broader context of how the developmental processes responsible for acquiring proper architecture of $\pi_{A}$ though the application of $\pi_{B}$ is not addressed here. This topic has been discussed in some detail in [1].

\section{Calculating variety of a human agent and its Umwelt}

The problem addressed here is funded in Ashby's law of requisite variety ${ }^{[7]}$. This law states that for a successful control of a system, the system that controls has to have at least as many states as the system that is being controlled. Otherwise, it would not be possible to produce a sufficient number of different responses, given all the number of different challenges that the environment poses on a controller, or on an agent in general. The question is then how many states can a human brain (or an AI-agent) theoretically assume and is this number sufficiently large to address the variety of the real-life problems that such agents face? The immediately following questions is: Can an increase in the number of policies (traverses) improve any limitations in the variety of the agent?

The key presumption behind the present calculations is that the upper limit of the total variety of states that a policy of an agent can produce is limited by the total amount of memory that the policy requires. The available amount of memory represents the maximum entropy that the system can generate and yet that its actions are informed about the environment in which it acts. Therefore, although one may argue that an agent could produce high entropy simply by generating noise, relevant for satisfying the law of requisite variety is only behavior that is informed about the properties of environment. The latter follows from the good regulator theorem ${ }^{[8]}$, which states that a regulator can be successful in regulating a system only if it is a good model of that system. The memory requirements on variety are the memory requirements for becoming a good model of the world in which the agent operates.

The calculation of total variety gives an upper bound estimate of what an agent can perform, how many different sensory inputs it can distinguish in order to consider making different actions. If one thinks of agent's memory as a set of templates against which the input is matched, then the estimate of total memory is related to an estimate of the number of templates that could be used by that agent.

In other words, we ask the question of how many different patterns (templates) can the brain store in its (synaptic) memory. As we will see, we are looking at the brain more as something like a read-only memory (ROM), rather than a memory that can easily replace its contents. The reason for that is that it takes for us a lifetime to acquire knowledge about the world and once acquired, this knowledge is not easily replaceable. The number of those patterns acquired through lifetime indicates the maximum variety of states that a developed brain, as an Ashby's regulator, can generate for the agent in order to produce meaningful actions on the environment to help with agent's survival. In other words, this number indicates: 1) the amount of knowledge that the brain can possibly have on how to respond in a given situation and by doing so, 2) the variety of responses to sensory inputs that it can produce based on that knowledge.

We are interested only in meaningful informed states, i.e., states that reflect some previously acquired knowledge about the surrounding world. Of course, the molecules in the brain can have many more states, but if these additional states are not stimulus-dependent, they either have to be mutually dependent (correlated) or have to be understood as noise.

We are considering here first agents that do not learn (only later we consider learning). Traditional brain theory and traditional AI both rely on two-traverses (i.e., T2agents). One of these traverses is for learning, which is the adaptive mechanism located lower on the practopoietic 
hierarchy. The other traverse is implemented by the mechanisms for processing inputs and executing actions, usually referred to as neural activity in brain sciences and as policies in machine learning. We are interested in the variety of this single top-level traverse. In contrast to the traditional $\mathrm{T} 2$-agents, the novel approach with T3-agents presumes the use of the two highest-level traverses for processing inputs and executing actions ${ }^{[1]}$.

The question addressed presently is whether T2-agents can possibly generate sufficient variety of behavior in reallife situations or whether instead only a T3-agent can satisfy those needs. So far, T2-agents have been implemented (either as a brain theory or as an AI) to limited domains of problems, which require much less variety than what an average adult human person may need in real life. The present question is whether these T2-approaches can scale up to the real human-level demands and thus to humanlevel intelligence.

\subsection{Variety generated by human brain}

For an average adult human brain without further learning, we can estimate the total variety based on the total number of synapses and the amount of bits stored at each synapse. According to a recent study, an optimistic estimate is that a single synapse can store 4.6 bits of information ${ }^{[9]}$. Furthermore, if there are about 1000 synapses for each neuron and there are about 100 billion neurons in the brain, we have in total $10^{11} \times 1000=10^{14}$ or 100 trillion synapses. We can now compute two types of variety, one for a brain that has already matured and cannot make substantial changes easily (i.e., it cannot suddenly replace its memories with memories of another person), and the other for all possible sets of lifelong experiences that a mature brain could potentially encounter (all different lives that a person could theoretically live).

Given that 4.6 bits of information can be stored per synapse $^{[9]}$, this would set the upper bound of the total theoretical variety that a mature adult human brain can generate without further learning to

$$
4.6 \times 10^{14} \text { bits. }
$$

As 4.6 bits can assume a total of $2^{4.6}=24.25$, which is about 24 states, we have a total of about

$$
2.4 \times 10^{15} \text { different states. }
$$

This is roughly 500 terabytes of memory, and is within the realms of what can be achieved by today's IT technology.

It is first important to note this number indicates the total variety of input-output that an adult brain can generate and that it corresponds to total memory storage.

A different calculation is for the total number of possible states that the memory can assume. In that case, the total number of possible states should be calculated as the number of states at a synapse, $2^{4.6}=24$ to the power of the number of synapses, i.e.,

$$
\left(24^{10}\right)^{14}
$$

which is a much larger number (even larger than the number of atoms in the visible universe, which is only about $10^{80}$ ).

So, why would we not consider the latter number as the theoretical limit of the variety that a human brain can produce? The difference between numbers calculated in (2) and (3) is the difference between what an adult brain can produce once it has gone through the development, education, etc. as opposed to the variety of all kinds of different developments and educations that a person may have gotten. The number in (3) is the variety of all possible lives that a person could theoretically live. However, once we have lived one life, the specific set of experiences defines what we have acquired in our long-term memory, and that knowledge cannot be changed any longer, or at least not easily (in a way, it acts as a ROM). And this is what number (2) describes. In the present paper, we are concerned with (2): We are considering the number of states a trained brain can generate, without having an option to live another life with a different set of experiences. According to the above estimate, this variety is limited to only about $10^{15}$ states. $^{1}$

The number $10^{15}$ gives us the maximum number of different responses that a mature adult brain can create without further learning. To imagine what this number may indicate, consider the famous patient H.M. who at adult age lost the ability to create new long-term memories after bilateral medial temporal lobectomy ${ }^{[10]}$. H.M. retained all his previous knowledge and was perfectly able to hold a conversation, read text, watch a movie or interact with his environment. He only could not create any new longterm memories. He could not change his knowledge up to the point of the surgery but could perfectly use the previously acquired knowledge. The question is then: How many different stimuli, sentences, events, situations could H.M. distinguish and respond to meaningfully? According to the above calculation and a T2-theory of the brain, this number is $4.6 \times 10^{14}$ bits and indicate the total richness of his mental life that could possibly occur.

In other words, by freezing learning, we are turning a $\mathrm{T} 2$-agent into a T1-agent, albeit well trained. This number tells us how many input-output mappings can be maximally preformed.

What the real task behind processing a sensory input is

\footnotetext{
${ }^{1}$ The difference between the two types of varieties can be illustrated if we consider a medium for memory storage such as DVDROM. If a DVD has 4.7 GB of memory, there are two different types of variety we can calculate. One is the number of different movies that can be stored on that DVD. This number corresponds to the calculation made in (3) and is a huge number (also, much larger than the number of atoms in the universe). The second variety is the amount of data that can be red from the memory storage once a movie has been burned on the DVD. This number is much smaller. It is only $4.7 \mathrm{~GB}$. We are considering this smaller number in the present study.
} 
for H.M. (or any other intelligent adaptive agent) is to compare inputs with the entire existing knowledge of all possible patterns that it can detect. In a simple pattern recognition task, the agent has to identify the stimulus against its entire database. And we humans can do this very well immediately. For example, we can just see a car by checking the shape in the stimulus against all of the other shapes that we have in the memory.

This direct distinguishability of stimuli at the perceptual level for human mind can be tested in experiments with perceptual pop-out ${ }^{[11,12]}$. These experiments tell us that we have also limitations. We are not able to distinguish any set of random stimuli, e.g., "IOVGJIZGSIOHIO" versus. "IOVGJIKGSIOHIO" cannot be distinguished without a slow serial search for a difference. However, either of the two sequences above can be easily distinguished from "IOVGJI_SIOHIO". The underscore symbol induces a socalled perceptual pop-out. Any every-day visual scene is full of perceptual pop-outs for a human mind.

However, what we humans really excel at in comparison to machines is that we are able to combine this variety of perceptual stimuli with the variety of semantic information. We test everything in parallel, the picture and its meaning. If the only problem of AI was finding the difference between two visual stimuli, a simple search algorithm would do that job and would by far outperform any human.

It turns out that there is a way to estimate how much information we are able to process in parallel. Our ability to process semantic information in parallel is related to the size of our working memory (or short-term memory). This memory storage is highly limited in capacity ${ }^{[13,14]}$, is highly correlated to intelligence quotient (IQ) ${ }^{[15]}$ and is based on semantic information extracted from long-term memory ${ }^{[13,14,16]}$. Our ability to store information in working memory is determined by how much knowledge we already possess about the stimuli. A color expert will be able to store more information about colors than a non-expert. An educated Chinese speaking person will be able to store more Chinese characters than a non-Chinese speaker, etc.

And humans are generally outperforming today's AI. We humans can solve many AI-related problems immediately i.e., much like H.M. could, without a need for additional learning. We could just look at a visual scene or just hear a narration and extract much more relevant information than an existing AI-machine can today. We use these simultaneous detection capabilities to make decisions while driving a car, watching a movie, or understanding language, and making purchasing decisions. In all those acts, we compare the current stimulus with all our knowledge acquired until that point in time - and we do it in a blink of an eye.

Thus, the high demands on the variety for an AI-agent come from this parallel template matching against the entire knowledge of the agent. These human capabilities of performing such matching processes fast make us smarter than the machine.

Our superiority is seen most obviously in situation in which the variety of sensory inputs has to be combined with the variety of semantics. This efficient combination of sensory+semantic contents makes us much better in understanding visual scenes and natural speech, or simply in playing the game of go (until recently).

The present analysis is about the question of whether $10^{15}$ provides sufficient storage for the patterns that the brain needs for such pattern-matching analyses. We compare two different theories of how the brain is adaptively organized (T2 versus T3 organization) with the estimates of the variety demands posted by the real life of an adult human person.

The present analyses are made under the assumption that all $10^{15}$ combinations are used without any redundancies or other sub-optimalities. Thus, we are estimating the maximal theoretical limits of pattern-matching mechanisms presuming that those have been implemented in the most optimal way possible.

\section{$2.2 \quad$ Variety of real life}

This number $10^{15}$ seems large for producing a lot of intelligent behavior, but the question is: Is it large enough? The other side of the equation is: How much variety does the real life require?

The question of the variety in the real life can be approached by calculating the amount of meaningful variety in sensory inputs to agents. We do not want to estimate the total number of combinations that pixels of an image can assume. We are interested only in the number of combinations that need to be understood by the agent in order to behave successfully in a given world. The question is how many different situations may a human observer need to distinguish, understand and respond to meaningfully? This would be then an estimate of how much variety the human brain should be able to account for.

In the first step of analysis, we focus only on the number of different sentences that a human mind may need to be able to comprehend. Our language is generative and a person may expect from the surrounding world any possible message, and should be thus able to decode any of them. To make a rough estimate of the order of magnitude of combinations that can emerge, let us presume that an educated native speaker of English has 15000 words in a vocabulary ${ }^{[17,18]}$. In addition, let us presume that adverbs, adjectives, verbs and nouns correspond respectively to $5 \%$, $20 \%, 20 \%$ and $55 \%$ of the vocabulary. This leads to 750 , $3000,3000,8250$ words in each of the four categories for an average speaker.

From those numbers, we can calculate the number of all combinations of sentences of different lengths. For threeword sentences that consists of a noun, followed by a verb and ending with a noun, we obtain roughly: $8250 \times 3000$ $\times 8250$

$$
\approx 2 \times 10^{11}
$$

combinations. 
This number fits within the variety of the human brain estimated above. But if we add an adjective to each of the nouns to make five-word sentences: adjective-noun-verbadjective-noun, we get a total of $2 \times 10^{11} \times 3000 \times 3000$

$$
\approx 2 \times 10^{18}
$$

combinations.

This number is already bigger than the limit that is posed by the total number of synapses in the brain, presuming that synapses are indeed the storage of information and that each synapse can store about 5 bits. If we add an adverb to each verb, the number of combinations grows even further, etc. Therefore, there seems not to be enough memory in our brains to generate a different response for five-word or larger sentences.

Importantly, however, we have to consider which of these sentences are meaningful to an average human, and which are not. One sentence that is not meaningful cannot be necessarily considered as producing a different brain state as another sentence that is also not meaningful. Rather, all meaningless sentences may be considered to result in one the same state (e.g., a "meaningless sentence" state). Indeed, most likely, majority of the sentences in the above calculation can be considered as not being successfully processed by human semantic machinery and hence as meaningless. To illustrate that point, we list here a few randomly generated sentences (from http://watchout4snakes.com/wo4snakes/Random/Random Sentence):

"The agony damages the regional spur below a pride."

"Our insult prices the flame."

"Behind the younger textbook quibbles an implied dealer."

Hence, only a small fraction of five-word sentences should be counted as meaningful. But there are also many more six-word, seven-word long and longer sentences that humans find perfectly meaningful. Each of these meaningful sentences should induce a different state in the brain. Thus, the total number of possible meaningful sentences is not easy to estimate. Below, we make this assessment based on the capacity of human working memory.

Before that, let us first point out that T2-theories presume that both sensory and semantic processing are performed at the same level of organization and thus, that it is not just the meaning that the brain (or an AI) needs to account for. It is the also the sensory inputs. All of those functions are covered by the number $4.6 \times 10^{14}$ bits.

This means that the above calculations suggest that a human brain should be unable to distinguish already at the sensory level most of five-word sentences (let alone their meaning). As the discrepancy is not small but is almost four orders of magnitude, this would mean that most of the pairs of random five-word sentences a brain with the variety of $4.6 \times 10^{14}$ bits could not be even noticed as two different sentences.

In other words, if we simulate on a computer an artificial neural network with 100 billion neurons, 1000 synapses per neuron and 4.6 bits per synapse, the network would not be large enough to associate a different response for each of the possible five-word sentences but could only do it for three-word sentences.

If the properties of this network correspond to the capacities of our brain, we also could not distinguish most pairs of five-word sentences. Those pairs should sound the same to us if pronounced, or look the same if written on a paper.

But this is clearly not the case. For us, it is easy to distinguish such sentences. How is that possible?

Before addressing this question and discussing the properties of T3-agents, let us first note that the combinatorial problem of the real world versus the limited variety of a brain, does not stop at language. The problem is the same and becomes possibly even bigger when vision is considered. Vision may require even larger variety than language both at the level of semantics and at the level of sensory inputs. Visual objects have different colors, sizes, shapes, positions, shades, etc.

When trying to understand the variety of processing in vision, we can ask a question of how many meaningful visual scenes our brain is capable of perceiving and distinguishing? To estimated that number, we will turn to the capacity of visual working memory (a.k.a. short-term memory). Working memory is not just a storage of information. It is a place where information is processed and this processing/storage depends primarily on the meaningfulness of the items ${ }^{[16,19]}$. Working memory stores information by the very means of finding meaning in it ${ }^{[13]}$. Hence, the capacity of working memory can be used as an indicator of how much meaning can visual system extract from a visual scene.

Experiments indicate that visual working memory can store about four objects ${ }^{[20,21]}$ and only if we are very familiar with them ${ }^{[14,19-21]}$ and only if a category exists for each object $^{[16]}$. Thus, if we conservatively assume that an adult human is able to distinguish 10000 different categories of objects, working memory for four objects would require a total variety of

$$
\left(10^{4}\right)^{4}=10^{16}
$$

combinations. This would mean that already the combinations needed for visual working memory cannot be accounted for by the memory of $10^{15}$ states. Working memory capacity reflects human capacity to understand a visual scene and is tightly related to the attentional capacity ${ }^{[19,22,23]}$. The present result would mean that the variety provided by our synaptic memory is not sufficient to enable us to understand a visual scene of four objects.

One possibility is that a T2-brain has more capacity to generate variety than the currently estimated. Another possibility would be that the capacity of four is an overestimate and involves some type of chunking (as shown for tasks that show capacity larger than four ${ }^{[14]}$ and that the "true" capacity of visual working memory is perhaps just three objects. The latter hypothesis would lead to

$$
\left(10^{4}\right)^{3}=10^{12}
$$


combinations, and would fit well within the supposed $10^{15}$ combinations of a T2-brain. Therefore, similarly to what we have concluded for the semantics of verbal materials, the semantic properties of visual working memory may fit with some stretching(!) - to the apparent limits of the brain.

However, even if both of the above hypotheses were correct and the brain had in the same time more storage than assumed (e.g., more synapses) and the working-memory capacity of three objects, not four, still another source of a combinatorial problem would remain. The above calculation accounts only for the semantic memory, i.e., object identities, and does not take into account the variety of sensory inputs with which these objects come. ${ }^{[20]}$ The fact is that there is not a single shape, size, color or shading for most of the objects that we can recognize and categorize. Normally, visual objects come in a huge variety of visual appearances and this variety needs also to be taken care of by the brain.

If we conservatively assume that we can perceptually easily detect each object in just 10000 different forms, this leads to

$$
\left(10^{12}\right)^{4}=10^{48}
$$

combinations for three-object working memory (attention capacity), and to

$$
\left(10^{16}\right)^{4}=10^{64}
$$

combinations for four objects. These numbers exceed readily the estimated capacity of the brain.

In fact, the number of visual combinations in which visual objects can come and can be perceptually distinguished by our visual system without any significant effort may be even larger. If we just assume that we can perceive an object, e.g., a car, in 10 different shapes, in 10 sizes of retinal projections and in 10 orientations, with 10 different colors, and 10 patterns of shading, we already have $10^{5}$ combinations for that object. And these numbers are likely to be much higher in reality. A similar problem holds for auditory inputs and recognition of speech.

These real-life variety numbers seem too high to be accounted for by stretching the estimates of the number of synapses or their individual memory capacity. Rather, it seems that there is a fundamental discrepancy between what a T2-brain of reasonable size can offer (be it biological or not) and what the real-life demands pose on human-level intellectual capabilities.

In conclusion, it seems that the T2-theory of the brain, which bases mental operations on a single policy, may account for the total variety of semantics, but the problem is with the additional variety of perceptual inputs. It seems that the combinatorial possibilities of perceptual inputs in real life create the real problem as they need to combine with semantics and the resulting variety exceed by far what a maximally optimized brain with 100 billion neurons and 1000 synapses per neuron could possibly deal with.

\subsection{Variety of T3-agents}

The above problems have been encountered when a single policy was considered. Here, we will discuss how multiple policies can provide a relief for that problem (called variety relief in practopoietic theory $\left.{ }^{[1]}\right)$. To understand the solution offered by the variety relief in T3-agents, it is useful to first consider the boost in variety that can be achieved by the process of learning in a T2-agent. If learning is not frozen and thus, we presume a full healthy brain (not H.M.'s brain), we can repurpose the resources and replace one type of knowledge that is no longer needed, with new knowledge that may be more valid in a new situation. That way, when learning is allowed, a much higher total variety can be produced.

For example, if memory storage for some text-storage device is limited to just one million characters, only one or a few books can be stored in this memory. However, if the device can "relearn" by deleting old and loading new books, the device can store all possible books that do not exceed 1 million characters. In fact, the total possible variety of that memory storage for is $10^{156}$ of different combinations of 26 letters in English alphabet (for comparison, as mentioned, the number of atoms in the visible universe is about $\left.10^{80}\right)$.

With a limited brain size or neural-network size, changes to the network's knowledge are thus the key process for boosting variety.

But what if not only the slow learning of facts and skill boosts variety, but in addition another mechanism operates and makes the brain change its knowledge at another level and at high speed. If the brain would have some quick way of reorganizing its anatomy and changing its memories, it could produce a much higher variety than $10^{15}$. It may have in fact enough variety to account for the richness of the sensory inputs.

The hierarchy of policies in a T3-agent described above in (1) can offer exactly this learning-based boost in variety. As policy $\pi_{A}$ can change policy $\pi_{N}$, and the total variety of the agent increases.

The speed of this adaptive change at $\pi_{A}$ would correspond to the "speed" of our thought. Each time we think a new thought or create a new mental image, we may be reorganizing our brains in such a fast way. Hence, the speed of this change should correspond to the speed of our mental processes, the lower limit being known to lay somewhere at 100 or 150 milliseconds ${ }^{[1]}$.

\subsection{How much can the variety increase theoretically?}

We have seen that maximum possible variety of a 1-million character storage is $10^{156}$, and this puts the upper bound as it presumes the "learning" mechanism (i.e., the loading mechanism), that is itself unlimited in knowledge creation capabilities. However, in most cases, this is not realistic. The learning mechanism has its own limitations. 
In general, when the variety of the learning mechanism is considered, the combined variety across two levels of organization can be computed as a product of the two varieties: If $\pi_{A}$ has $\mathrm{N}_{A}$ possible states, and $\pi_{N}$ has $\mathrm{N}_{N}$ states, the maximum total theoretical number of states that could be produced by the combined agent is

$$
\mathrm{N}_{A} \times \mathrm{N}_{N} .
$$

For example, in the 1-million character memory from the example above, we may presume a book-loading "learning" mechanism that has only 10 different states. This loading mechanism cannot load more than 10 different books. As a consequence, the total possible variety of the entire system (memory + loader) is 10 million different states. In general, depending on the limitations of the learning mechanisms, there will be normally a stark reduction in the number of combinations in comparison to what would be achieved by an unlimited learning mechanism (in the above example, $10^{7}$ down from $\left.10^{156}\right)$.

In adaptive systems, the limitations on learning come from the limited sources of knowledge. If the knowledge would be already prepared in a ready-to-use form and stored elsewhere, it could be simply loaded (like from a larger harddisk to the smaller RAM memory of a computer). This would make the problem trivial. Unfortunately, adaptive systems do not have such an auxiliary depository of knowledge of how to interact with the world. Rather, biological systems have to extract that knowledge from the environment, which is why they are adaptive on the first place.

As mentioned, the process of extracting knowledge from the environment is referred to as traverse in [1]. For example, application of reinforcement learning is a traverse, knowledge on how to learn stored at a lower level is applied through interaction with the environment in order to create new knowledge (new policy) at a higher level. Hierarchy of policies in (1) generalizes that relation.

\subsection{How many states can a frozen T3- brain theoretically produce?}

Let us presume that the brain is a T3-agent and that when frozen (i.e., without learning), it becomes a T2-agent. Let us also presume that the brain uses much of its variety for the lower level of the two remaining, i.e., for storing $\pi_{A}$. This is where the abstract knowledge is stored such as concepts. Hence, this level of organization can be referred to as ideatheca (meaning storage of concepts).

Let us conservatively assume that ideatheca (i.e., $\pi_{A}$ ) has just $10^{12}$ states, which is what we estimated above as the lower bound of semantic capacity enabling three-item working-memory. Next, let us presume that $\pi_{N}$ has even less variety and set it to the value $10^{10}$. This presumes that only a small portion of the entire brain's resources is under the influence of ideatheca and can be changed quickly in less than a second. In particular, the choice of this number presumes that only $1 / 1000$-th of the total memory machinery of the brain is being changed in such a rapid way.
Under these assumptions, the total number of states that a combined $\pi_{A} \rightarrow \pi_{N}$ could produce without any additional learning is

$$
10^{12} \times 10^{10}=10^{22}
$$

This number is much larger than $10^{15}$ and much more suitable for coping with the estimated real-life requirements on variety. This number indicates that if H.M. was a T3agent before the surgery and became limited to a T2-agency after the surgery (losing his third traverse), this patient may have had the possible richness of mental life that could deal with $10^{22}$ combinations. Irrespective of whether the estimates of his semantic memory of concepts is about $10^{12}$ or $10^{16}$, there is still a lot of room left for additional combinations of sensory inputs that indicate those concepts in the surrounding world and that H.M. could efficiently process.

The number $10^{22}$ would also correspond to a neural network that has 100 billion neurons and 1000 synapses per neuron, but also has an additional set of mechanisms that change the properties of the network with a rapid rate and on the basis of the incoming sensory inputs. To achieve variety of $10^{22}$, it would be sufficient to enable changing one bit of information per neuron (there are about 100 billion neurons in human brain). For example, a neuron could be switched on or off by its adaptation mechanisms.

For this to work, a pre-requirement is that the slow learning mechanisms noted as $\pi_{G}$ in (1) provide the knowledge to $\pi_{A}$ on how to adjust $\pi_{N}$. In other words, by slow learning mechanisms and throughout many years of the development of the nervous system, the network must first learn how to make these quick adjustments to its $\pi_{N}$. That is the network has to acquire the $10^{12}$ amount of $\pi_{A}$ knowledge through its development time.

In that case, the agent can be considered as "understanding" the sensory inputs. Understanding would mean that the operation of $\pi_{A}$ gives the stimuli best possible interpretation given all of the knowledge that the agent has acquired through lifetime (for details see the section on abductive reasoning in tri-traversal agents in [1]).

The alternative to extending the hierarchy would be to cope with the variety requirements by simply increasing the total size of the given policy, i.e., by increasing the network size. In that case, variety grows linearly with the number of components. To double the variety of patterns stored in the brain, the size of the brain needs to be doubled. To increase variety to $10^{22}$ states, from $10^{15}$ states in a 1.5 -kilogram brain, we would need an increase to $1.5 \times 10^{7}$ kilograms of biological mass. This is more than the cumulative size of all the brains of all the people currently living on planet Earth.

\section{Conclusions}

A T2-AI, which means an AI based on single memory storage and on a single set of learning mechanisms, cannot possibly reach the intelligence of human. This conclusion 
is made on the basis of Ashby's requisite variety theorem ${ }^{[7]}$ and an estimate of the total theoretical variety of control that a brain can create given the number of neurons and synapses. It turns out that the variety the brain could possibly create if it was a T2-agent would not be enough to deal successfully with the demands of the real-world environment. However, we also show that if the organization of the brain formed a T3-agent, the size of the brain would suffice. Accordingly, an AI that would mimic human intelligence would have to be organized as a T3-agent too.

The variety of a T2-agent would be sufficient to implement all of the semantic knowledge of an adult human person that can be expressed in words, but would not suffice for the requirements of the sensory processing of those semantic categories. The objects and situations that need to be detected from the sensory data, require too much variety to be dealt with a T2-brain. This is the case for both recognition of visual scenes and understanding of speech, and the problem does not go away even if the coding and processing is maximally optimized in the brain.

The important implication of the present analysis is that no novel optimization or invention of a new algorithm, or discovery of a new architecture for neural circuits can possibly bring a T2-agent (i.e., a traditional single-policy + learning-mechanism agent) with reasonable size of resources to a human-level intelligence. The present calculations already presume that all the operations and coding schemes in the organization of the agent have been optimized to the theoretical maximum. Thus, no new creative invention in machine learning is possible that could bring to the intelligence level of humans the modern approaches to AI. In other words, to build artificial general intelligence, we need to seek beyond deep learning networks, Markov chains or Bayesian networks and similar. Otherwise, we would need to scale up the resources to prohibitively large sizes.

The only way to create an artificial system that is humanlevel intelligent with reasonable resources is to implement a hierarchy of policies, which then makes possible the decisions about driving, walking, moving, etc. to rely on the full variety of the sensory data. A T3-agent with realistic computational resources can perform such a task and, once it has acquired knowledge of an average adult person, it could generate variety of $10^{22}$ states. This number is sufficient to deal with all the semantic knowledge and still plenty of sensory information can be processed. And, if needed, there would be enough room for increasing that number within the realm of the current IT technology.

A change from $\mathrm{T} 2$ to $\mathrm{T} 3$-organization comes with some $\operatorname{costs}^{[1]}$. One cost is that the entire agent operates always slower with more than with fewer traverses. This is because the additional adaptive processes require time to complete. In human mental operations, this slowdown ranges from $100 \mathrm{~s}$ of milliseconds to seconds (for more details see [1]).

In [1], it has been proposed that the physiological mechanism underlying anapoiesis, i.e., the application of knowledge in ideatheca to change network properties, are im- plemented through neural adaptation. Furthermore, these mechanisms are proposed to rely on sensory inputs and hence, largely on the variety stored in synapses that process those sensory inputs. In [24], several testable empirical predictions have been proposed as derived from the theorized T3-organization of the brain.

In conclusion, an AI that matches human intellectual capabilities is possible only in tri-traversal systems.

\section{Acknowledgments}

The author would like to thank Hrvoje Nikolić, Raul C. Muresan, Shan Yu and Matt Mahoney for valuable comments on previous versions of the manuscript.

Open Access This article is distributed under the terms of the Creative Commons Attribution 4.0 International License (http://creativecommons.org/licenses/by/4.0/), which permits unrestricted use, distribution, and reproduction in any medium, provided you give appropriate credit to the original author(s) and the source, provide a link to the Creative Commons license, and indicate if changes were made.

\section{References}

[1] D. Nikolić. Practopoiesis: Or how life fosters a mind. Journal of Theoretical Biology, vol. 373, pp. 40-61, 2015.

[2] D. Nikolić. Practopoiesis: How cybernetics of biology can help AI. [Online], Availabe: https://www.singularityweblog.com/practopoiesis/, 2014

[3] G. R. Chen. Pinning control and controllability of complex dynamical networks. International Journal of Automation and Computing, vol. 14, no. 1, pp. 1-9, 2017.

[4] Y. Jiang, J. Y. Dai. An adaptive regulation problem and its application. International Journal of Automation and Computing, vol. 14, no. 2, pp. 221-228, 2017.

[5] R. S. Sutton, A. G. Barto. Reinforcement Learning, Cambridge, Mass, USA: MIT Press, 1998.

[6] C. J. C. H. Watkins. Learning from Delayed Rewards, Ph. D. dissertation, Cambridge University, UK, 1989.

[7] W. R. Ashby. Principles of the self-organizing dynamic system. The Journal of General Psychology, vol. 37, no. 2, pp. 125-128, 1947.

[8] R. C. Conant, W. R. Ashby. Every good regulator of a system must be a model of that system. International Journal of Systems Science, vol. 1, no. 2, pp. 89-97, 1970.

[9] T. M. Bartol, C. Bromer, J. P. Kinney, M. A. Chirillo, J. N. Bourne, K. M. Harris, T. J. Sejnowski. Hippocampal spine head sizes are highly precise. bioRxiv, [Online], Available: http://dx.doi.org/10.1101/016329, March 11, 2015.

[10] S. Corkin. Lasting consequences of bilateral medial temporal lobectomy: Clinical course and experimental findings in H.M. Seminars in Neurology, vol. 4, no. 2, pp. 249-259, 1984 . 
[11] A. M. Treisman, G. Gelade. A feature-integration theory of attention. Cognitive Psychology, vol. 12, no. 1, pp. 97-136, 1980.

[12] A. Treisman. Preattentive processing in vision. Computer Vision, Graphics, and Image Processing, vol. 31, no. 2, pp. 156-177, 1985.

[13] G. A. Miller. The magical number seven plus or minus two: Some limits on our capacity for processing information. Psychological Review, vol. 63, no. 2, pp. 81-97, 1956.

[14] N. Cowan. The magical number 4 in short-term memory: A reconsideration of mental storage capacity. Behavioral and Brain Sciences, vol. 24, no. 1, pp. 87-114, 2001.

[15] R. W. Engle, M. Kane, S. W. Tuholski. Individual differences in working memory capacity and what they tell us about controlled attention, general fluid intelligence, and functions of the prefrontal cortex. Models of Working Memory: Mechanisms of Active Maintenance and Executive Control, A. Miyake, P. Shah, Eds., Cambridge, USA: Cambridge University Press, pp. 102-134, 1999.

[16] H. Olsson, L. Poom. Visual memory needs categories. Proceedings of the National Academy of Sciences of the United States of America, vol. 102, no. 24, pp. 8776-8780, 2005.

[17] A. Cervatiuc. Highly Proficient Adult Non-native English Speakers' Perceptions of their Second Language Vocabulary Learning Process, Ph. D. dissertation, University of Calgary, Canada, 2007.

[18] P. Nation, R. Waring. Vocabulary size, text coverage and word lists. Vocabulary: Description, Acquisition and Pedagogy, N. Schmitt, M. McCarthy, Eds., Cambridge, USA: Cambridge University Press, pp. 6-19, 1997.

[19] G. A. Alvarez, P. Cavanagh. The capacity of visual shortterm memory is set both by visual information load and by number of objects. Psychological Science, vol. 15, no. 2, pp. 106-111, 2004.

[20] S. J. Luck, E. K. Vogel. The capacity of visual working memory for features and conjunctions. Nature, vol. 390, no. 6657, pp. 279-281, 1997.

[21] D. Nikolić, W. Singer. Creation of visual long-term memory. Perception \& Psychophysics, vol. 69, no. 6, pp. 904-912, 2007.

[22] E. Awh, J. Jonides. Overlapping mechanisms of attention and spatial working memory. Trends in Cognitive Sciences, vol. 5, no. 3, pp. 119-126, 2001.
[23] J. S. Mayer, R. A. Bittner, D. Nikolić, C. Bledowski, R. Goebel, D. E. J. Linden. Common neural substrates for visual working memory and attention. Neuroimage, vol. 36, no. 2 , pp. 441-453, 2007.

[24] D. Nikolić. Testing the theory of practopoiesis using closed loops. Closed Loop Neuroscience, A. El Hady, Ed., Amsterdam: Academic Press, 2016.

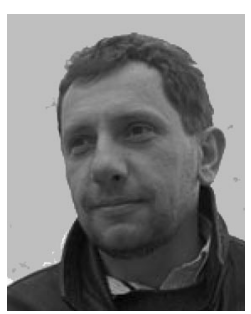

Danko Nikolić received the degree in psychology and a degree in civil engineering from the University of Zagreb, Croatia. He received the Master's degree and the $\mathrm{Ph} . \mathrm{D}$. degree in cognitive psychology from at the University of Oklahoma, USA. In 2010, he received a Private Docent title from the University of Zagreb, and in 2014 an associate professor title from the same university. He is now associated with Frankfurt Institute for Advanced Studies and works at DXC Technology in the field of artificial intelligence and data science.

He has a keen interest in addressing the explanatory gap between the brain and the mind. His interest is in how the physical world of neuronal activity produces the mental world of perception and cognition. For many years he headed an electrophysiological lab at Max-Planck Institute for Brain Research. He approached the problem of explanatory gap from both sides, bottom-up and top-down. The bottom-up approach begins from brain physiology. The top-down approach investigates the behavior and experiences. Each of the two approaches led him to develop a theory: The work on behavior and experiences led to the discovery of the phenomenon of ideasthesia (meaning "sensing concepts"). The work on physiology resulted in the theory of practopoiesis (meaning "creation of actions").

He has conducted many empirical studies in the background of those theories. These studies involved simultaneous recordings of activity of 100+ neurons in the visual cortex (extracellular recordings), behavioral and imaging studies in visual cognition (attention, working memory, long-term memory), and empirical investigations of phenomenal experiences (synesthesia). His research was supported by grants from the Hertie Foundation, Deutsche Forschungsgemeinschaft (DFG) and other sources.

E-mail: danko.nikolic@gmail.com (Corresponding author) ORCID iD: 0000-0002-9317-8494 